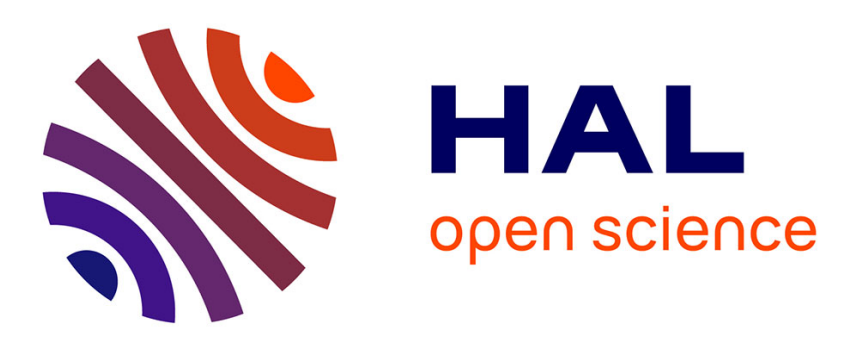

\title{
Diagnostic accuracy of microbial keratitis with in vivo scanning laser confocal microscopy
}

Scott Hau, John Dart, Minna Vesaluoma, Dipak Parmar, Ilse Claerhout, Kanom Bibi, Frank Larkin

\section{- To cite this version:}

Scott Hau, John Dart, Minna Vesaluoma, Dipak Parmar, Ilse Claerhout, et al.. Diagnostic accuracy of microbial keratitis with in vivo scanning laser confocal microscopy. British Journal of Ophthalmology, 2010, 94 (8), pp.982. 10.1136/bjo.2009.175083 . hal-00557354

\section{HAL Id: hal-00557354 \\ https://hal.science/hal-00557354}

Submitted on 19 Jan 2011

HAL is a multi-disciplinary open access archive for the deposit and dissemination of scientific research documents, whether they are published or not. The documents may come from teaching and research institutions in France or abroad, or from public or private research centers.
L'archive ouverte pluridisciplinaire HAL, est destinée au dépôt et à la diffusion de documents scientifiques de niveau recherche, publiés ou non, émanant des établissements d'enseignement et de recherche français ou étrangers, des laboratoires publics ou privés. 


\section{Diagnostic accuracy of microbial keratitis with in vivo scanning laser confocal microscopy}

Scott C Hau, ${ }^{1}$ John K G Dart, ${ }^{1}$ Minna Vesaluoma, ${ }^{1}$ Dipak N Parmar, ${ }^{2}$ Ilse Claerhout, ${ }^{3}$ Kanom Bibi, ${ }^{1}$ Daniel F P Larkin ${ }^{1}$

${ }^{1}$ NIHR Biomedical Research Centre in Ophthalmology, Moorfields Eye Hospital and Institute of Ophthalmology, London, UK, EC1V 2PD

${ }^{2}$ Dept of Ophthalmology, Whipps Cross Hospital, London, UK, E11 1NR

${ }^{3}$ Ghent University Hospital, De Pintelaan 185, 9000 Gent, Belgium

Word count: 2500

Corresponding author: Scott Hau, Research fellow, Moorfields Eye Hospital NHS

Foundation Trust, 162 City Road, London, UK, EC1V 2PD

Email: scott.hau@moorfields.nhs.uk

Tel: +447905 295888

Fax: +442075662471

Key words: Confocal microscopy, diagnostic accuracy, acanthamoeba keratitis, fungal keratitis, Microsporidia, Nocardia 
The Corresponding Author has the right to grant on behalf of all authors and does grant on behalf of all authors, an exclusive licence (or non-exclusive for government employees) on a worldwide basis to the BMJ Publishing Group Ltd and its Licensees to permit this article (if accepted) to be published in British Journal of Ophthalmology and any other BMJPGL products to exploit all subsidiary rights, as set out in our licence 


\title{
ABSTRACT
}

\begin{abstract}
Aims
To determine the accuracy of diagnosing microbial keratitis by masked medical and non-medical observers using the Heidelberg Retina Tomograph II / Rostock Cornea Module in vivo confocal microscope.
\end{abstract}

\section{Methods}

Confocal images were selected for 62 eyes with culture or biopsy proven infections. The cases comprised of 26 Acanthamoeba, 12 fungus, 3 Microsporidia, 2 Nocardia, and 19 bacterial infections (controls). The reference standard for comparison was a positive tissue diagnosis. These images were assessed on two separate occasions by 4 observers who were masked to the tissue diagnosis. Diagnostic accuracy indices, Kappa (К) statistic and percentage agreement values were calculated. The Spearman correlation coefficient $\left(\mathrm{r}_{\mathrm{s}}\right)$ was calculated for the number of correct diagnoses versus duration of disease.

\section{Results}

The highest sensitivity and specificity values were $55.8 \%$ and $84.2 \%$, and the lowest sensitivity and specificity values were $27.9 \%$ and $42.1 \%$. The highest positive and negative likelihood ratios were 2.94 and 0.59, respectively. Agreement values were: fair to moderate $(\kappa, 0.22-0.44)$ for reference standard versus observer diagnosis, moderate to good in intra-observer variability (repeatability, $\kappa ~ 0.56-0.88$ ), and poor to moderate in inter-observer variability (reproducibility, $\kappa, 0.15-0.47$ ). The correct 
diagnosis was associated with duration of disease for Acanthamoeba keratitis $\left(\mathrm{r}_{\mathrm{s}}=0.60\right.$, $\mathrm{p}=0.001)$

\section{Conclusions}

The diagnostic accuracy of microbial keratitis by confocal microscopy is dependent on observer experience. Intra-observer repeatability was better than inter-observer reproducibility. Difficulty in distinguishing host cells from pathogenic organisms limits the value of confocal microscopy as a stand-alone tool in diagnosing microbial keratitis. 


\section{INTRODUCTION}

2

3 Difficulties in clinical and microbiological diagnosis are one of the major problems in

4 the management of microbial keratitis particularly when caused by protozoa

5 (Acanthamoeba and Microsporidia), fungi or filamentary bacteria. Diagnosis of these

6 pathogens is difficult as they often take days or weeks to grow in culture and, in any

7 case, culture is insensitive with culture positive rates rarely exceeding 60\%.[1]

8 Although culture is still the primary diagnostic tool in tertiary referral centres it is not

9 widely available to many patients because of limited resources.

10

11 The confocal microscope allows detailed in vivo analysis of normal[2] and

12 pathological corneas. In patients with presumed corneal infection, it is used in

13 diagnosis and in examination of the extent of involvement of tissue by infection and

14 associated inflammation. All published studies have been directed at diagnosis and a

15 number have shown both white light and laser confocal microscopy to be effective in

16 diagnosing Acanthamoeba,[3-5] fungal,[6-8] Nocardia[9] and Microsporidia

17 keratitis.[10] However, these studies only present case series or reports and there are

18 limited published data on evaluating the diagnostic accuracy of confocal microscopy.

19 Two recent studies have found high sensitivity and specificity values for diagnosing

20 fungal keratitis (FK) and Acanthamoeba keratitis (AK) with the Confoscan 3.0 (Nidek

21 Technology, Padova, Italy).[11,12] However, factors such as observer or selection

22 bias, the absence of masking the observers from the microbiological diagnosis, and

23 lack of appropriate controls may have resulted in overestimates of the sensitivity and

24 specificity values. Although experience in interpreting confocal keratitis images is

25 essential, the accuracy of diagnosing microbial keratitis by clinicians with differing 
levels of confocal microscopy experience and the potential of using trained technicians in interpreting images have not previously been assessed. These are important considerations in evaluating this technique. The aim of this study was to examine the diagnostic accuracy of microbial keratitis with the Heidelberg Retina Tomograph II / Rostock Cornea Module (HRT II/RCM) in vivo confocal microscope, as a stand-alone tool, by trained medical and non-medical observers with differing confocal microscopy experience.

\section{MATERIALS AND METHODS}

\section{Patients}

This study was approved by the Research \& Ethics Committee of Moorfields Eye Hospital and it adhered to the tenets of Declaration of Helsinki. We retrospectively reviewed the case notes of a consecutive series of microbial keratitis patients who had had both corneal cultures or corneal biopsy and confocal microscopy $(n=105)$ from January 1, 2005 to January 4, 2008. These cases were both those refractory to conventional treatment and those with unusual clinical features such as perineural infiltrates and ring infiltrates. Patients were either referred from Moorfields Emergency Department or from other institutions. Of the 105 cases, 62 culture or biopsy positive cases (62 eyes) were identified: 26 Acanthamoeba, 11 fungus, 1 fungus and bacteria, 3 Microsporidia, 2 Nocardia, and 19 bacteria. Bacteria were used as controls because they are normally too small to detect with confocal microscopy, $[9,13]$ therefore the case which was culture positive for both fungus and bacteria was classified as a fungal keratitis for the purposes of the study. We did not classify 
Nocardia as controls because they are filamentous bacteria and can form filamentous structures that are large enough to be distinguished by confocal microscopy.[9] Empirical treatments started prior to assessment in this study included topical antimicrobial agents and topical steroids for presumed herpes, bacterial or keratitis of unknown cause, respectively. Irrespective of the referring diagnosis, all patients had undergone a full clinical examination by a corneal specialist and repeat corneal scraping for culture and confocal microscopy on the same day. If the scraping was culture negative, and the keratitis progressive, then a corneal biopsy was later performed. Exclusion criteria were culture or biopsy negative keratitis cases, and patients who declined to have confocal microscopy or a corneal culture as part of their clinical investigation. The reference standard for this study was a diagnosis either by isolation on culture of a corneal scraping or histological diagnosis on a corneal biopsy; other ancillary culture sources such as contact lens case and solutions were not used.

The clinical outcomes were recorded for all the patients in the study and were consistent with the diagnosis based on culture or histology therefore it is unlikely, but possible, that there was unrecognised polymicrobial infections which may have been identified on confocal but not by culture or biopsy. We followed the Standards for Reporting of Diagnostic accuracy (STARD) initiative in conducting this study.[14]

\section{Culture and biopsy methods}

Corneal scrapings for microbial culture were inoculated on the following media: blood agar, Sabouraud's dextrose agar (fungi), Robertson's cooked meat (anaerobic bacteria), Escherichia coli-seeded non-nutrient agar (Acanthamoeba), brain heart infusion (fastidious organisms, fungi) and Lowenstein-Jensen (mycobacteria, Nocardia). Scrapings were smeared on sterile glass slides for Gram and Giemsa stains. 
75 All microbiological investigations were undertaken independently in an external laboratory. For biopsy a superficial lamellar disc of the affected cornea was trephined under local anaesthetic to provide a further specimen for microbiology and histopathological staining.

\section{Confocal microscopy measurement protocol}

In vivo confocal microscopy was performed on all 62 eyes by a single experienced observer (SH) with the HRT II / RCM (Heidelberg Engineering GmbH, Dossenheim, Germany) confocal microscope following a Standard Operating Procedure as follows. A sterile Tomocap (Heidelberg Engineering GmbH, Dossenheim, Germany) was mounted over the objective of the microscope (Zeiss, x 63), and Polyacrylic acid $0.2 \%$ (Viscotears, Novartis) was used as a coupling agent between the cap and the lens objective. Topical anaesthetic (Proxymetacaine hydrochloride $0.5 \%$, Chauvin) and Carmellose sodium 1\% (Celluvisc, Allergan) was instilled into both eyes to provide comfort and act as a coupling fluid between the front of the Tomocap and the cornea. Options for image acquisition include section (a single image at a particular depth), volume (a series of images over $60 \mu \mathrm{m}$ depth) and sequence scans (a video sequence at a particular depth). The volume scan option was selected for image acquisition because it allowed the capturing of large number of images over a short space of time. The central region of the corneal ulcer or corneal infiltrate was scanned first followed by the top, left, bottom and right margin of the lesion. At each point, the epithelial layer of the affected area was scanned first and the focal plane of the microscope adjusted until the whole depth of the ulcer or infiltrate had been scanned. When there was more than one infiltrate, the same scanning sequence was repeated for each infiltrate. The wavelength of the laser employed in the HRT II / RCM is $670 \mathrm{~nm}$ and 
100

101

102

103

104

105

106

107

108

113 corneal ulcer or infiltrate was selected and exported onto Microsoft Power Point ${ }^{\circledR}$

114 (Microsoft Corp., Redmond, WA, USA). These included those of Acanthamoeba -

115 round single or double walled hyper-reflective objects $(\sim 10-20 \mu \mathrm{m})$ consistent with

116 Acanthamoeba cysts,[4,5] fungus - linear irregular branching hyper-reflective objects

117 consistent with fungal hyphae,[6,7] Microsporidia - small round hyper-reflective

118 deposits $(\sim 2 \mu \mathrm{m})$ located in between keratocytes,[10] Nocardia - small branching

119 filamentous structures within the corneal stroma,[9] and bacteria (control) - a mixture

120 of inflammatory cells. 
125 All digital images were assessed prospectively in the same standard fashion in the

126 Reading Centre at Moorfields Eye Hospital by 4 observers ( 3 ophthalmologists and 1

127 medical technician) with differing levels of experience in assessing keratitis on

128 confocal microscopy as follows. Of the 3 ophthalmologists, observer A had 6 years of

129 experience in assessing microbial keratitis with confocal microscopy, observer B, 10

130 years of experience in confocal microscopy but not keratitis, and observer C, 6

131 months of experience in assessing keratitis with confocal microscopy. Observer D

132 was a medical technician who had 2 years of experience in performing confocal

133 microscopy using the HRT II / RCM and analysing keratitis images but with no

134 experience in the clinical appearance and treatment of different types of keratitis. To

135 ensure each observer was familiar with the image appearance of different cell types

136 obtained from the HRT II / RCM confocal microscope, examples of both normal

137 cellular morphology and the standard images of different pathogens were shown in a

138 presentation before their assessment. In addition, a series of five recent articles on

139 diagnosing keratitis with the HRT II / RCM $[4,5,7,9,10]$ were given to each observer

140 to read 2 weeks prior to their scheduled assessment date.

142 The confocal images were viewed in random order and assigned an identification

143 number from 1 to 62 . To ensure that there was masking between observers, the order

144 of viewing the images were randomised by computer before being assessed by the

145 next observer on a different day. No clinical details regarding each case were made

146 available to the observers. Each observer assessed the series of images in a masked

147 fashion on slide show in Microsoft Powerpoint ${ }^{\circledR}$ and recorded the diagnosis

148 corresponding to one of the following categories: AK, FK, Microsporidia (MK), 
149 Nocardia (NK) or bacterial keratitis (BK). A reference sheet showing the range of

150 sizes of resident and inflammatory cells including epithelium and macrophages, and

151 pathogenic cells e.g. diameter of Acanthamoeba cysts was given to each observer.

152 Intra-observer variability (repeatability) was evaluated by asking each observer to

153 reassess the images, randomised in a different order, three weeks later in the same

154 standard fashion. Inter-observer variability (reproducibility) was assessed by

155 determining the level of agreement in diagnosis between observers. Readings of all

156 the digital images were collected on a standard pro-forma and analysed.

\section{Data analysis}

159 Data analysis was performed with SPSS V14.0 (SPSS Inc, Chicago, USA). We

160 calculated sensitivity, specificity, positive and negative likelihood ratios (LR) for both

161 image set for each observer. Positive LR predicts the probability of a positive test

162 result in patients with disease compared to those who do not have the disease.

163 Negative LR predicts the probability of a negative test in those who have the disease

164 compare to those who do not. The level of agreement between the reference standard

165 and different observers, and both intra and inter-observer variability were determined

166 using Kappa ( $\kappa)$ statistic. The interpretation of $\kappa$ statistic is as follows: 'poor' if $\kappa \leq$

1670.20 , 'fair' if $\kappa 0.21-0.40$, 'moderate' if $\kappa 0.41-0.60$, 'substantial' if $\kappa 0.61-0.80$

168 and 'good' if $\kappa>0.80$.[16] In addition, we also calculated percentage agreement

169 values between reference standard and observers, within-observers, and between

170 different observers. Spearman's rank correlation coefficient $\left(\mathrm{r}_{\mathrm{s}}\right)$ was used to determine

171 the relationship between the number of correct diagnoses and the duration of disease

172 for AK, FK and BK respectively. The duration of disease was defined as the time

173 from symptom onset to presentation to the Corneal and External Disease Service at 
174 Moorfields. A value of $\mathrm{P}<0.05$ was deemed statistically significant. MK and NK

175 were excluded from this analysis because the numbers were too small.

176

177 RESULTS

178

179 The reference standard consisted of 52 culture positive cases from corneal scrapings

180 and 10 histopathologically confirmed cases on corneal biopsy. Sensitivity, specificity

181 and likelihood ratio values for each observer are shown in Table 1.

\begin{tabular}{|c|c|c|c|c|}
\hline Observer & Sensitivity (95\% CI) & Specificity $(95 \%$ CI) & Positive LR (95\% CI) & Negative LR (95\% CI) \\
\hline \multicolumn{5}{|l|}{$\mathrm{A}$} \\
\hline 1st assessment & $55.8(47.7-63.2)$ & $57.9(39.5-74.7)$ & $1.32(0.79-2.49)$ & $0.76(0.49-1.33)$ \\
\hline 2nd assessment & $55.8(47.6-61.8)$ & $73.7(55.0-87.3)$ & $2.12(1.06-4.87)$ & $0.60(0.44-0.95)$ \\
\hline \multicolumn{5}{|l|}{$\mathrm{B}$} \\
\hline 1 st assessment & $53.4(45.4-58.8)$ & $78.9(60.5-91.0)$ & $2.53(1.15-6.51)$ & $0.59(0.45-0.90)$ \\
\hline 2nd assessment & $46.5(38.6-50.9)$ & $84.2(66.4-94.2)$ & $2.94(1.15-8.82)$ & $0.64(0.52-0.93)$ \\
\hline \multicolumn{5}{|l|}{$\mathrm{C}$} \\
\hline 1st assessment & $27.9(20.5-32.3)$ & $84.2(67.5-94.2)$ & $1.77(0.63-5.56)$ & $0.86(0.72-1.18)$ \\
\hline 2nd assessment & $27.9(20.4-33.2)$ & 78.9 (61.9-90.9) & $1.32(0.54-3.66)$ & $0.91(0.74-1.29)$ \\
\hline \multicolumn{5}{|l|}{$\mathrm{D}$} \\
\hline 1st assessment & $44.2(36.8-52.3)$ & $42.1(25.3-60.5)$ & $0.76(0.49-1.33)$ & $1.33(0.79-2.49)$ \\
\hline 2nd assessment & $48.8(40.7-56.3)$ & $57.9(39.6-74.8)$ & $1.16(0.67-2.23)$ & $0.88(0.59-1.50)$ \\
\hline \multicolumn{5}{|c|}{$\begin{array}{l}95 \% \mathrm{CI}=95 \% \text { confidence intervals; } \mathrm{LR}=\text { likelihood ratio } \\
\text { Positive LR = the probability of a positive test result in patients with disease compared to those who do not have the } \\
\text { disease. } \\
\text { Negative } \mathrm{LR}=\text { the probability of a negative test result in patients with disease compared to those who do not have the } \\
\text { disease. }\end{array}$} \\
\hline
\end{tabular}

183 The highest sensitivity value obtained was $55.8 \%$ and the highest specificity value

$18484.2 \%$. We found fair to moderate agreement between observers and reference

185 standard $(\kappa, 0.22-0.44)$, moderate to good agreement in intra-observer variability $(\kappa$,

$1860.56-0.88)$, and poor to moderate agreement in inter-observer variability $(\kappa, 0.15-0.47)$, 
187 Table 2. One observer (observer B) obtained the highest positive and lowest negative

188 LR for diagnosing microbial keratitis. This observer also achieved the best overall

189 kappa and percentage agreement values in diagnoses compared to reference standard,

190 Table 3. The best inter-observer agreement (percentage agreement, 61.3-66.1\%; $\kappa$,

191 0.43-0.47) was between observer A and B, the two most experienced observers in the

192 study. Observer $\mathrm{C}$ was the most repeatable (percentage agreement, 93.5\%; $\kappa, 0.88$ )

193 despite having the lowest kappa and percentage agreement values compared to

194 reference standard, Tables $2 \& 3$.

195

Table 2. Kappa values: reference standard versus observers, intraobserver and inter-observer variability

\begin{tabular}{llllll}
\hline Observer & Reference standard & $\mathrm{A}$ & $\mathrm{B}$ & $\mathrm{C}$ & $\mathrm{D}$ \\
\hline $\mathrm{A}$ & $0.36(0.42)$ & 0.56 & $0.43(0.47)$ & $0.23(0.15)$ & $0.28(0.29)$ \\
\hline $\mathrm{B}$ & $0.44(0.40)$ & $0.43(0.47)$ & 0.76 & $0.40(0.32)$ & $0.18(0.32)$ \\
\hline $\mathrm{C}$ & $0.24(0.22)$ & $0.23(0.15)$ & $0.40(0.32)$ & 0.88 & $0.24(0.33)$ \\
\hline $\mathrm{D}$ & $0.27(0.36)$ & $0.28(0.29)$ & $0.18(0.32)$ & $0.24(0.33)$ & 0.65 \\
\hline
\end{tabular}

Reference standard $=$ culture or biopsy proven cases

Values in parentheses = observers' second assessment

196

197

\begin{tabular}{|c|c|c|c|c|c|}
\hline & $\begin{array}{l}\text { Table 3. Percentag } \\
\text { standard and obse } \\
\text { observers. }\end{array}$ & $\begin{array}{l}(\%) \text { agree } \\
\text { ers, within }\end{array}$ & $\begin{array}{l}\text { ent values b } \\
\text { bservers, al }\end{array}$ & $\begin{array}{l}\text { tween refer } \\
\text { I between c }\end{array}$ & $\begin{array}{l}\text { lce } \\
\text { ferent }\end{array}$ \\
\hline Observer & Reference standard & $\bar{A}$ & $\mathrm{~B}$ & $\mathrm{C}$ & $\mathrm{D}$ \\
\hline A & $56.5(61.3)$ & 70.9 & $61.3(66.1)$ & $45.2(43.5)$ & $45.2(50)$ \\
\hline B & $61.3(58.1)$ & $61.3(66.1)$ & 85.5 & $64.5(61.3)$ & $37.1(53.2)$ \\
\hline$\overline{\mathrm{C}}$ & $45.2(43.5)$ & $45.2(43.5)$ & $64.5(61.3)$ & 93.5 & $40.3(53.2)$ \\
\hline $\mathrm{D}$ & $43.5(51.6)$ & $45.2(50)$ & $37.1(53.2)$ & $40.3(53.2)$ & 72.6 \\
\hline \multicolumn{6}{|c|}{ Reference standard = culture or biopsy proven cases } \\
\hline
\end{tabular}

198 
200 Complete agreement in diagnosis between all the observers and reference standard for

201 both assessments were found in 3/26 (11.5\%) cases of AK, 8/19 (42.1\%) cases of BK,

202 and 1/12 (8.3\%) case of FK. In contrast, none of the observers identified

203 Acanthamoeba in 5/26 (19.2\%) cases, fungus in 4/12 (33.3\%), and confused BK with

204 other diagnoses in 2/19 (10.5\%) cases. Observer B was the only one who managed to

205 diagnose NK correctly in one case. The percentage correct diagnosis for the different

206 types of keratitis is shown in Table 4. A breakdown of all the diagnoses for each

207 observer for the different keratitis category is shown in the appendix.

208

Table 4. Percentage of correct diagnoses of the different causes of keratitis for different observers

\begin{tabular}{|c|c|c|c|c|}
\hline \multirow{2}{*}{$\begin{array}{l}\text { Reference standard } \\
\text { Diagnosis }\end{array}$} & \multicolumn{4}{|c|}{ Observer, n (\%) } \\
\hline & Observer A & Observer B & Observer $\mathrm{C}$ & Observer D \\
\hline Acanthamoeba $(\mathrm{n}=26)$ & $19(73.1)$ & $11(42.3)$ & $4 \quad(15.4)$ & $12(46.2)$ \\
\hline Bacteria* $(n=19)$ & $10(52.6)$ & $15(78.9)$ & $15(78.9)$ & $8 \quad(42.1)$ \\
\hline Fungus $^{\dagger}(\mathrm{n}=12)$ & $1(8.3)$ & $5 \quad(41.2)$ & $5 \quad(41.2)$ & $5 \quad(41.2)$ \\
\hline $\operatorname{Microsporidia}(\mathrm{n}=3)$ & $1 \quad(33.3)$ & $2 \quad(66.7)$ & $2(66.7)$ & $1 \quad(33.3)$ \\
\hline Nocardia $(\mathrm{n}=2)$ & 0 & $1 \quad(50)$ & 0 & 0 \\
\hline
\end{tabular}

Percentage correct diagnosis was calculated by taking into account only those cases where each observer had made the correct diagnosis on both assessments.

* 2 Enterobacter, 1 Haemophilus, 1 Moraxella, 1, Mycobacteria, 1 Pasteurella, 3 Pseudomonas, 1 Serratia, 7 Staphylococcus, 2 Streptococcus.

${ }^{\dagger} 1$ Alternaria, 1 Candida, 10 Fusarium.

209

210 Figure 1 shows a series of images demonstrating the difference in appearance between

211 correctly diagnosed versus incorrectly diagnosed cases. Figures $1 \mathrm{~A}$ and F show a

212 case of late diagnosed AK versus early diagnosed AK; note the presence of

213 inflammatory cells in the epithelium in early AK making distinction between host

214 cells and Acanthamoeba cysts and trophozoites difficult, whereas in delayed

215 diagnosed AK, single or clusters of cysts were seen in the stroma with minimal host

216 immune and resident cells seen. The incorrectly diagnosed cases demonstrate the 
217 difficulties in distinguishing host cells from pathogenic organisms, and Nocardia (Fig

218 1J) from FK because of their similarity in appearance on confocal microscopy.

220 Figure 2 shows a plot between the number of correct diagnoses for Acanthamoeba, 221 bacteria and fungal keratitis versus the duration of disease (days). The graph shows a

222 moderate correlation between the number of correctly diagnosed cases and the

223 duration of disease for $\mathrm{AK}\left(\mathrm{r}_{\mathrm{s}}=0.60, \mathrm{p}=0.001\right)$, but not for $\mathrm{BK}\left(\mathrm{r}_{\mathrm{s}}=0.17, \mathrm{P}=0.49\right)$ or

224 FK $\left(r_{s}=-0.19, p=0.57\right)$, respectively. Therefore, the longer the duration of AK, the 225 higher the likelihood that a correct diagnosis was made by the observers in grading 226 the confocal images.

\section{DISCUSSION}

230 Acanthamoeba and fungus are uncommon causes of corneal infection for which early 231 diagnosis is paramount because it yields better prognosis and reduces ocular 232 morbidity.[17,18] Although the current reference standard for diagnosing microbial 233 keratitis is corneal culture, the sensitivity varies because of numerous factors.[19]

235 The HRT II / RCM in vivo confocal microscope has been shown to be useful in 236 diagnosing a range of pathogens but validation studies of this new technology are few. 237 A recent review has reported the efficacy of diagnosing infections keratitis with 238 confocal microscopy to be inconclusive, with the possible exception of AK.[20] Our 239 results show moderate sensitivity and moderate to high specificity values in 240 diagnosing microbial keratitis with the HRT II / RCM confocal microscope, whereas 
241 both Kanavi et al[11] and $\mathrm{Tu}$ et al[12] found very high sensitivity (>90\%) in

242 diagnosing AK and FK respectively with the Confoscan 3. Tu et al,[12] using multi-

243 test referencing standards, reported that when there are both clinical characteristics

244 and objective evidence of AK, the adjunctive usage of confocal microscopy exhibited

245 a sensitivity of $90.6 \%$ and specificity of $100 \%$. In our study, we set out to evaluate the

246 diagnostic accuracy of confocal microscopy as a stand alone tool rather than a

247 supportive investigative technique, without the bias and influence of clinical findings.

248 Although assessing confocal images in the absence of clinical data does not reflect the

249 use of confocal microscopy in clinical settings, it is the only way to avoid bias when

250 analysing the images. Our inclusion criteria were based on culture positive cases

251 irrespective of confocal classification. Although we chose only one representative

252 image from each case this was the best available image for the organism that was

253 cultured from each case giving the observers the best opportunity to make a correct

254 confocal diagnosis; we believe that reviewing a series of images from each case

255 would either have made a correct confocal diagnosis more difficult or have had no

256 effect on the outcome. In addition, it allowed standardisation when viewing the

257 images so that all observers assessed the same number of images consecutively. The

258 absence of controls in the previous studies and the use of confocal 'positive' without

259 culture confirmation as a reference standard, or for the case definition[11,12], could

260 lead to selection bias and misdiagnosis resulting in an overestimation of sensitivity

261 values.[12,21,22] This is evident from our controls in which immune cells can often

262 be confused with AK cysts and vice versa leading to erroneous diagnosis.

263 Furthermore, 'good' confocal images have been illustrated in most published studies

264 to present findings without discussion of difficulties in analysing equivocal images.

265 We found fair to moderate agreement between reference standard and observer 
266 diagnosis when a case mix of equivocal and unequivocal images were analysed by our

267 observers. The rigorous criteria in our study design in regard to the use of masked

268 observers and controls could explain why sensitivity values, even for the most

269 experienced observer, were lower.

270

271 Another explanation for the very high sensitivity values reported in one previous

272 study was the use of only one ophthalmology trained observer who, in addition to

273 being unmasked to the clinical findings, was experienced in the use of confocal

274 microscopy for keratitis diagnosis: this makes it difficult to extrapolate the results to

275 what might be expected outside their centres.[12] To evaluate the potential of using

276 this technology in clinics where an ophthalmologist with experience in confocal

277 microscopy may not be available, our graders included 2 experienced

278 ophthalmologists, an inexperienced ophthalmologist and an experienced technician.

279 We found a two-fold difference in sensitivity between the most experienced and the

280 least experienced observer indicating higher diagnostic accuracy with clinicians

281 experienced in confocal microscopy. Our results indicate the sensitivity value with a

282 trained technician, with no experience in the clinical appearance of different types of

283 microbial keratitis, was better than an inexperienced medical observer but with a

284 lower specificity value and positive LR. This raises the possibility of training non-

285 medical personnel, in performing and analysing keratitis images. The highest positive

286 LR and lowest negative LR was achieved by observer B who was experienced in

287 confocal imaging of normal corneal anatomy and various pathological conditions

288 other than microbial keratitis, indicating experience gained in other aspects of

289 confocal microscopy improves the diagnostic outcome. 
291 Intra-observer agreement (repeatability) was found to be moderate to good, indicative

292 of good observer repeatability in grading the images irrespective of the accuracy of

293 their diagnoses. Observer experience did not appear to improve intra-observer

294 repeatability as the observer with the lowest sensitivity had the highest repeatability

295 and vice versa. Inter-observer agreement (reproducibility) was poor to moderate

296 between different observers because of factors such as observer experience and

297 differences in techniques of classifying images by different observers. The two

298 observers who had the highest sensitivity values also had the best inter-observer

299 reproducibility, indicating experienced observers achieved a higher diagnostic

300 accuracy and reproducibility than less experienced observers. Therefore, to improve

301 reliability the same experienced operator should be employed if sequential imaging of

302 a patient is required.

303

304 Our observers were able to diagnose AK more accurately than any other type of 305 keratitis. The unique appearance of Acanthamoeba cysts on confocal microscopy and 306 the higher number of AK compared to other conditions in our study might explain this 307 outcome. However, AK was commonly confused with controls and vice versa because 308 of the diagnostic difficulty with some of the equivocal images. There was a marked 309 association between the accuracy of diagnosing AK and the duration of disease.

310 Previous case reports have mainly described the morphological features of cysts and 311 trophozoites in the epithelium and stroma during active infection,[4,5] but have not 312 related the number of cysts seen and the way they distribute with the different stages 313 of the disease process. In early disease, where the organism is mainly confined to the 314 epithelium, the presence of large numbers of inflammatory cells made diagnosing AK 315 more difficult because of the difficulty in distinguishing AK cysts and particularly 
316 trophozoites from inflammatory cells.[5] Late presentation was associated with either

317 a greater number of Acanthamoeba cysts seen in the images or the fact that they were 318 easier to identify because of a reduction in the type and number of host cells seen. Our 319 experience, therefore, suggests that AK is easier to identify with confocal microscopy 320 in the later stages of infection.

322 The use of confocal microscopy in diagnosing FK has been widely reported in the 323 literature.[6-8] Filamentous fungal hyphae have characteristic linear hyper-reflective 324 lesions branching at 45 or 90 degrees angle, [7] whereas candida infection produces 325 pseudofilaments.[7] Despite these well described confocal appearances of FK in the 326 literature, the percentage of correct diagnosis in our series was low possibly due to 327 difficulties in differentiating other linear images from fungal hyphae.[23]

329 Nocardia and Microsporidia species are rare causes of microbial keratitis.[24] 330 Clinically, Nocardia may be misdiagnosed as mycotic or mycobacterial keratitis, $[9,25]$ 331 whilst Microsporidia can be misdiagnosed as AK or herpes simplex keratitis. Despite 332 the rarity of these organisms, because of the unique appearance on confocal 333 microscopy with Microsporidia,[10] two observers managed to identify this organism 334 correctly in both of their assessments. Only observer B managed to obtain the correct 335 diagnosis in both assessments for diagnosing one case of Nocardia keratitis; the 336 unfamiliarity in interpreting confocal images of Nocardia, the similarity in appearance 337 of fungal hyphae and Nocardia filaments, and the small number of cases in our study 338 made diagnosing this organism difficult. The inclusion of both Nocardia and 339 Microsporidia cases might have reduced the overall sensitivity and specificity values 
340 but as confocal findings of both organisms have been reported, we believe it was

341 appropriate to include them in the study.

343 In summary, to the best of our knowledge, this is the first study evaluating the

344 diagnostic accuracy of microbial keratitis using a single reference standard for

345 different masked observers with the HRT II / RCM confocal microscope. Although

346 confocal microscopy is non-invasive and can provide a rapid diagnosis for microbial

347 keratitis, (i) similarities between inflammatory and pathogenic cells, and (ii) difficulty

348 in interpreting equivocal images, limits its usefulness as a stand-alone tool in

349 diagnosing keratitis. Confocal microscopy is a useful adjunct in managing refractory

350 cases and we have shown that the diagnostic accuracy improves with clinician

351 experience. However, the diagnostic accuracy of confocal microscopy used in

352 isolation from the clinical assessment is still too low to be a substitute for tissue

353 diagnosis, particularly in patients with progressive disease. Improvement in clinician

354 training and experience, greater standardization of image interpretation, and the

355 development of new software in tandem with higher resolution imaging is likely to

356 improve the diagnostic accuracy of this technology in diagnosing microbial keratitis

357 in the future.

358

359 ACKNOWLEDGEMENTS

360 The authors would like to thank Dr Catey Bunce for her statistical advice and support.

\section{COMPETING INTERESTS}

363 None declared 
FUNDING

366 None

367

368

369 REFERENCES

370

371 1. Yeh DL, Stinnett SS, Afshari NA. Analysis of bacterial cultures in infectious

372 keratitis, 1997 to 2004. Am J Ophthalmol 2006;142;1066-1068.

373

374 2. Jalbert I, Stapleton F, Papas E, et al. In vivo confocal microscopy of the human

375 cornea. Br J Ophthalmol 2003;87:225-236.

376

377 3. Parmar DN, Awward ST, Petroll WM, et al. Tandem scanning confocal corneal

378 microscopy in the diagnosis of suspected acanthamoeba keratitis. Ophthalmology $379 \quad 2006 ; 113: 538-47$.

380

381 4. Matsumoto Y, Dogru M, Sato E, et al. The application of in vivo confocal scanning

382 laser microscopy in the management of acanthamoeba keratitis. Molecular Vision $383 \quad 2007 ; 13: 1319-26$.

384

385 5. Kobayashi A, Ishibashi Y, Oikawa Y, et al. In vivo and ex vivo laser confocal

386 microscopy findings in patients with early-stage acanthamoeba keratitis. Cornea.

$387 \quad 2008 ; 27: 439-45$.

388 
6. Avunduk AM, Beuerman RW, Varnell ED, Kaufman HE. Confocal microscopy of

390 Aspergillus fumigatus keratitis. Br J ophthalmol 2003;87:409-410.

391

392 7. Brasnu E, Bourcier T, Dupas B, et al. In vivo confocal microscopy in fungal

393 keratitis. Br J Ophthalmol. 2007;91:588-91

394

395 8. Tu EY, Park AJ. Recalcitrant Beauveria bassiana keratitis: confocal microscopy

396 findings and treatment with posaconazole (Noxafil). Cornea 2007;26:1008-10.

397

398 9. Vaddavalli PK, Garg P, Sharma S, et al. Confocal microscopy for Nocardia

399 keratitis. Ophthalmology 2006;113:1645-50.

400

401 10. Sagoo MS, Mehta JS, Hau S, et al. Microsporidium stromal keratitis: in vivo

402 confocal findings. Cornea 2007;26:870-3.

403

404 11. Kanavi MR, Javadi M, Yazdani S, Mirdehghanm S. Sensitivity and specificity of 405 confocal scan in the diagnosis of infectious keratitis. Cornea 2007;26:782-6.

406

407 12. Tu EY, Joslin CE, Sugar J, et al. The relative value of confocal microscopy and 408 superficial corneal scrapings in the diagnosis of Acanthamoeba keratitis. Cornea $409 \quad 2008 ; 27: 764-72$

410

411 13. Petroll WM, Cavanagh HD, Jester JV. Clinical confocal microscopy. Curr Opin 412 Ophthalmol 1998;9:59-65. 
414 14. Bossuyt PM, Reitsma JB, Bruns DE, et al. Towards complete and accurate

415 reporting of studies of diagnostic accuracy: the STARD initiative. Standards for

416 Reporting of Diagnostic Accuracy. Clin Chem 2003;49:1-6.

417

418 15. Zhivov A, Stachs O, Stave J et al. In vivo three-dimensional confocal laser

419 scanning microscopy of corneal surface and epithelium. Br J Ophthalmol

$420 \quad 2009: 93: 667-672$.

421

422 16. Petrie A, Sabin C. Medical statistics at a glance. 2nd ed, Oxford: Blackwell

423 Publishing Ltd 2005: 93-95.

424

425 17. Bacon AS, Dart JK, Ficker LA, et al. Acanthamoeba keratitis. The value of early

426 diagnosis. Ophthalmology 1993;100:1238-43.

427

428 18. Duguid IG, Dart JK, Morlet N, et al. Outcome of acanthamoeba keratitis treated

429 with polyhexamethyl biguanide and propamidine. Ophthalmology 1997;104:1587-92.

430

431 19. McLeod SD, Kolahdouz-Isfahani A, Rostamian K, et al. The role of smears,

432 cultures, and antibiotic sensitivity testing in the management of suspected infectious

433 keratitis. Ophthalmology 1996;103:23-8.

434

435 20. Labbé A, Khammari C, Dupas B, et al. Contribution of in vivo confocal

436 microscopy to the diagnosis and management of infectious keratitis. Ocul Surf

$437 \quad 2009 ; 7: 41-52$.

438 
439 21. Gray R, Begg CB, Greenes RA. Construction of receiver operating characteristic

440 curves when disease verification is subject to selection bias. Med Decis Making

$441 \quad$ 1984;4:151-64.

442

443 22. Choi BC. Sensitivity and specificity of a single diagnostic test in the presence of

444 work-up bias. J Clin Epidemiol 1992;45:581-6.

445

446 23. Chiou AG, Kaufman SC, Beuerman RW, et al. Differential diagnosis of linear

447 corneal images on confocal microscopy. Cornea 1999;18:63-6.

448

449 24. Sridhar MS, Sharma S, Reddy MK, et al. Clinicomicrobiological review of

$450 \quad$ Nocardia keratitis. Cornea 1998;17:17-22.

451

452 25. Lalitha P, Tiwari M, Prajna NV, et al. Nocardia keratitis: species, drug

453 sensitivities, and clinical correlation. Cornea 2007;26:255-9.

454

455

456

457

458

459

460

461

462

463

464 
465

466

467

468

469

470

471

472

473

474

475

476

477

478

479

480

481

482

483

484

485

486

487

488

\section{FIGURE LEGENDS}

\section{Figure 1}

Confocal scans of correctly diagnosed versus incorrectly diagnosed cases.

Figures A to D demonstrate the characteristic features of inflammatory cells and pathogenic organisms on confocal microscopy in which all the observers had made the correct diagnoses. A, Acanthamoeba cysts (white arrow), some with doublewalled appearance (dotted white arrow). B, Inflammatory cells (black arrows). C, Fungal hyphae (black arrows). D, Microsporidia organisms (white arrows).

Figures $\mathrm{E}$ to $\mathrm{J}$ show a series of images of incorrectly diagnosed cases demonstrating the difficulty in distinguishing host cells from pathogenic organisms and Nocardia from fungal keratitis. E, Shows Nocardia filaments (white arrows) - only observer B identified this correctly with all the other observers graded it as fungal hyphae. F, Cultured Acanthamoeba - misdiagnosed as bacterial keratitis by observers B, C and D; possible Acanthamoeba cysts (white arrows), and possible inflammatory cells (black arrows). G, Cultured bacteria (Staphylococcus aureus) but was misdiagnosed as Acanthamoeba by all the observers; multiple round lesions that could be identified as inflammatory or Acanthamoeba cysts (white arrows). H, Cultured bacteria diagnosed as fungal keratitis by observers C \& D; linear hyphae-like opacities that were confused with fungal hyphae (white arrows). I, Cultured bacteria - diagnosed as Microsporidia by all the observers; small hyper-reflective granules that appear similar to Microsporidia organisms (white arrows). J, Cultured positive for Alternaria and 
489 Staphylococcus aureus but diagnosed as Nocardia by observers A, B and D; hyphae

490 type lesions that appear similar to Nocardia filaments (black arrows).

491

492 Figure 2

493

494 Scattered plot showing the relationship between number of correctly diagnosed cases

495 and duration of disease (days) for Acanthamoeba, bacteria and fungal keratitis.

496 


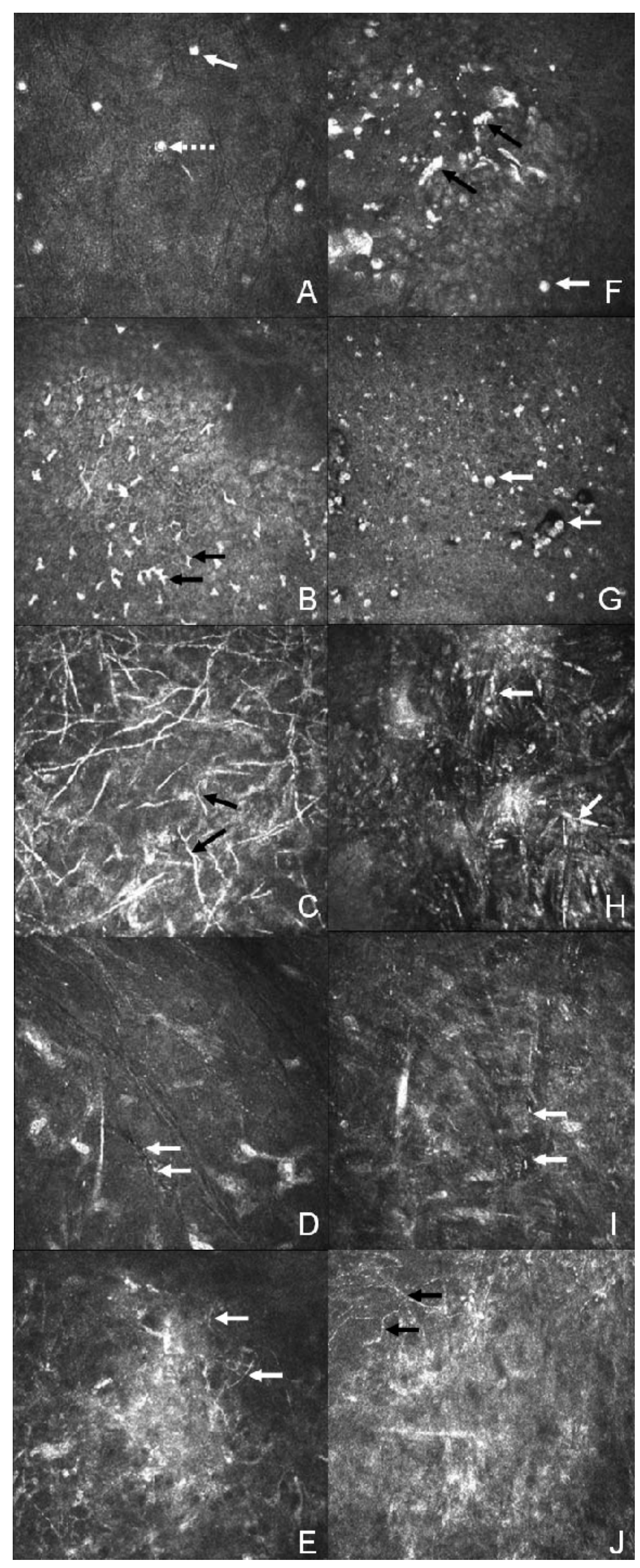




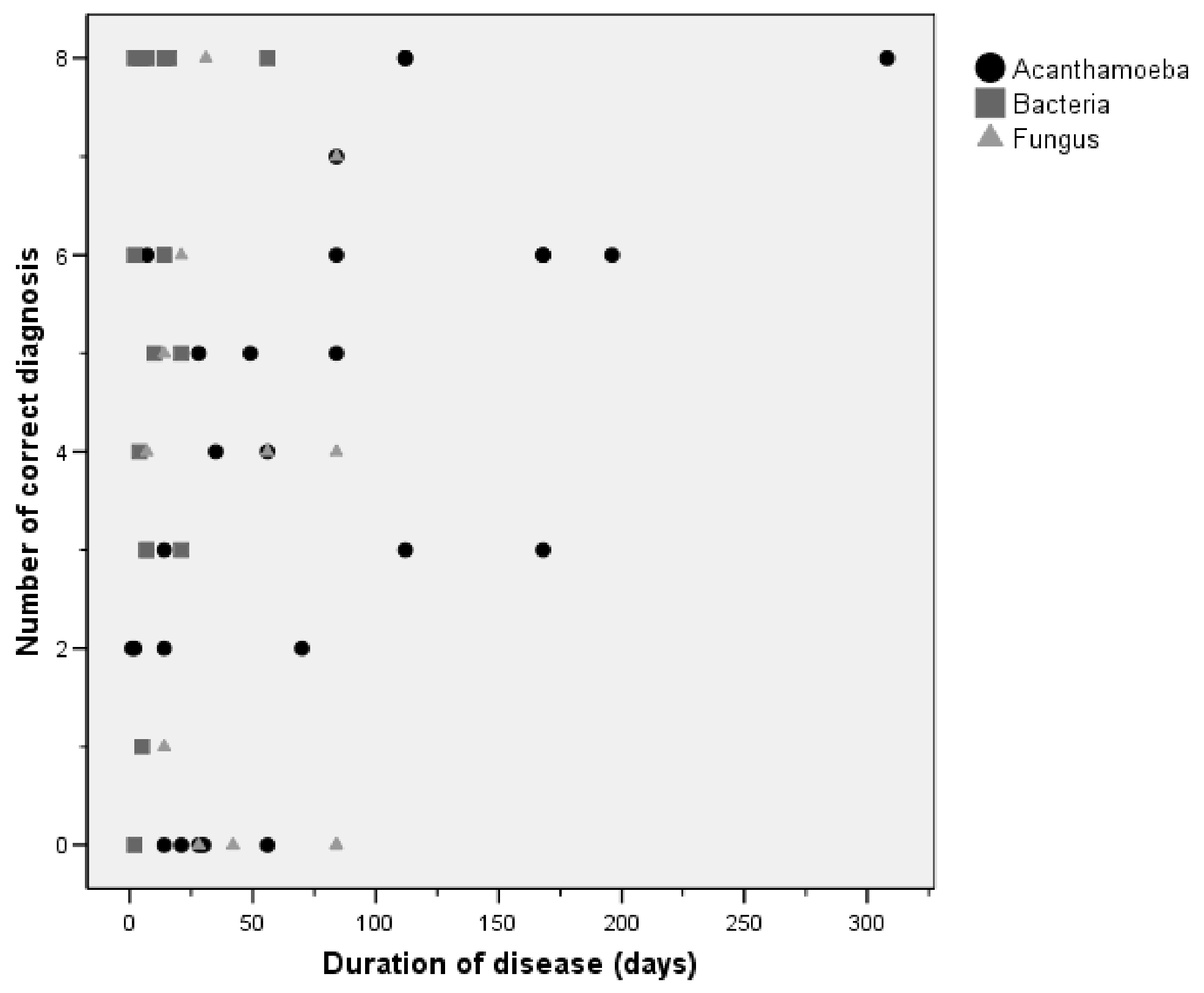

